\title{
g-modes in the late-type Be star $\beta$ CMi detected by the MOST satellite ${ }^{1}$
}

\author{
H. Saio, ${ }^{2}$ C. Cameron, ${ }^{3}$ R. Kuschnig, ${ }^{3}$ G. A. H. Walker, ${ }^{4}$ J. M. Matthews, ${ }^{3}$ \\ J. F. Rowe, ${ }^{3}$ U. Lee, ${ }^{2}$ D. Huber, ${ }^{5}$ W. W. Weiss, ${ }^{5}$ D. B. Guenther, ${ }^{6}$ A. F. J. Moffat, ${ }^{7}$ \\ S. M. Rucinski, ${ }^{8}$ D. Sasselov ${ }^{9}$ \\ ${ }^{2}$ Astronomical Institute, Tohoku University, Sendai, Japan \\ ${ }^{3}$ Dept. of Physics and Astronomy, University of British Columbia, Canada \\ ${ }^{4} 1234$ Hewlett Place, Victoria, BC V8S 4P7, Canada \\ ${ }^{5}$ Institut für Astronomie, Universität Wien, Austria \\ ${ }^{6}$ Dept. of Astronomy and Physics, St. Mary's University Halifax, Canada \\ ${ }^{7}$ Dépt. de physique, Univ. de Montréal, and Obs. du Mont Mégantic, Canada \\ ${ }^{8}$ Dept. of Astronomy \& Astrophysics, David Dunlap Obs., Univ. of Toronto, Canada \\ ${ }^{9}$ Harvard-Smithsonian Center for Astrophysics, Cambridge, MA 02138, USA
}

\begin{abstract}
The Microvariability and Oscillations of Stars (MOST) satellite has detected low-amplitude light variations $(\Delta m \sim 1 \mathrm{mmag})$ in the late-type $\mathrm{Be}$ star $\beta \mathrm{CMi}$ (B8Ve). The photometric variations have periods of $\sim 0.3$ days. This is consistent with high-order, prograde $(m=-1)$ g modes of a nearly critically rotating $3.5 M_{\odot}$ model.
\end{abstract}

\section{Results}

$\beta \mathrm{CMi}$ is one of the latest spectral type Be stars located near the cool boundary of the SPB instability region in the HR diagram (Pamyatnykh 1999). The MOST detection of multiple pulsations in $\beta \mathrm{CMi}$ is the first detection of photometric variations of this star and, more importantly, is the first detection of nonradial g modes in a Be star later than B6, leading to the possibility that pulsations are excited in all Be stars.

There are two significant frequency detections of 3.257 and $3.282 \mathrm{c} / \mathrm{d}$ and some marginal evidence for other frequencies (see Saio et al. 2007 for further details).

The top panel of Fig. 1 shows theoretical frequencies versus growth rates for the pulsations excited in a rapidly rotating $3.5 \mathrm{M}_{\odot}$ model. The model is from Saio et al. (2007); a slightly faster rotation rate is adopted to obtain better agreement with observations. In this model only prograde high-order $\mathrm{g}$ modes of $m=-1$ are excited by the $\kappa$-mechanism at the Fe-bump of opacity at $T \sim 2 \times 10^{5} \mathrm{~K}$. Since the frequencies of high-order $\mathrm{g}$ modes in the co-rotating frame are much smaller than the rotation frequency, the frequencies in the inertial frame group in a frequency range slightly higher than the rotation frequency. This phenomenon is typical of SPBe-type pulsations first observed in HD 163868 by Walker et al. (2005). In that case, an additional group of frequencies (for $m=-2$ ) appears around twice the rotation frequency. Figure 1 shows that the closely spaced frequencies observed in $\beta \mathrm{CMi}$ are consistent with high overtone $\mathrm{g}$ modes in a rapidly rotating star and that the identified frequencies lie in roughly the same frequency region as the theoretically excited $g$ modes.

\footnotetext{
${ }^{1}$ Based on data from the MOST satellite, a Canadian Space Agency mission, jointly operated by Dynacon Inc., the University of Toronto Institute of Aerospace Studies and the University of British Columbia with the assistance of the University of Vienna.
} 


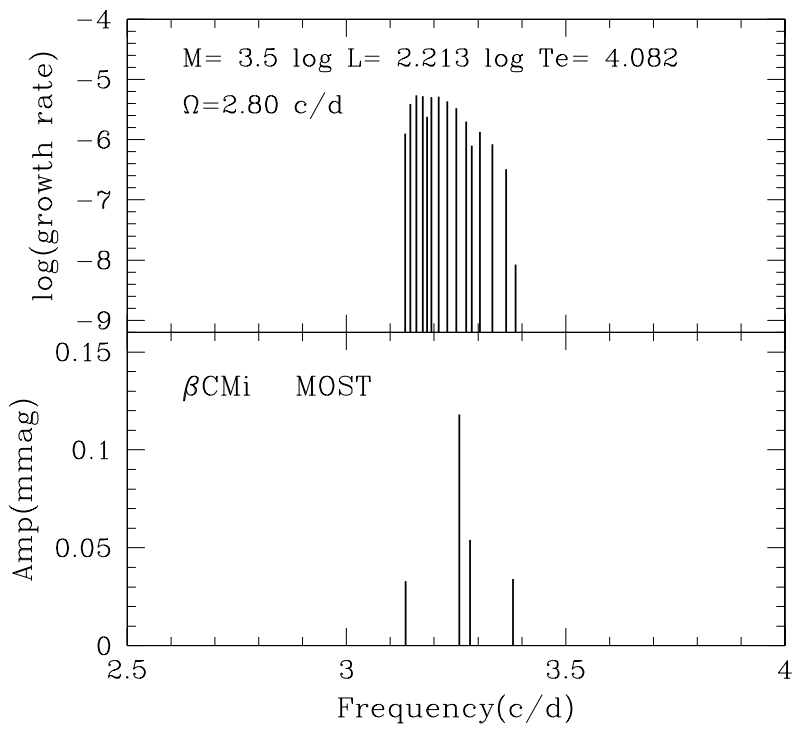

Figure 1: Observed frequencies versus amplitudes (bottom panel) and theoretical frequencies versus growthrates for excited prograde dipole $g$ modes of a rapidly rotating model (top panel).

\section{References}

Pamyatnykh A. A., 1999, Acta Astron., 49, 119

Saio H., Cameron C., Kuschnig R, et al., 2007, ApJ, 654, 544

Walker G. A. H., Kuschnig R., Matthews J. M., et al., 2005, ApJL, 635, L77 